\title{
Improved Stabilization Criteria for Neutral Time-Delay Systems
}

\author{
Lianglin Xiong, ${ }^{1,2}$ Haiyang Zhang, ${ }^{1,3}$ Yongkun Li, ${ }^{2}$ and Zixin $\mathrm{Liu}^{4}$ \\ ${ }^{1}$ School of Mathematics and Computer Science, Yunnan Minzu University, Kunming 650500, China \\ ${ }^{2}$ School of Mathematics and Statistics, Yunnan University, Kunming 650091, China \\ ${ }^{3}$ School of Science, Nanjing University of Science and Technology, Nanjing 210094, China \\ ${ }^{4}$ School of Mathematics and Statistics, GuiZhou University of Finance and Economics, Guiyang 550025, China \\ Correspondence should be addressed to Lianglin Xiong; lianglin_5318@126.com and Haiyang Zhang; haiya287@126.com
}

Received 23 February 2016; Revised 6 August 2016; Accepted 14 August 2016

Academic Editor: Haranath Kar

Copyright (C) 2016 Lianglin Xiong et al. This is an open access article distributed under the Creative Commons Attribution License, which permits unrestricted use, distribution, and reproduction in any medium, provided the original work is properly cited.

This paper addresses the stabilization conditions for neutral systems with mixed time delays. By constructing a novel class of Lyapunov functionals which contains an augmented Lyapunov functional, using a new class of improved Jensen's like inequalities, two improved delay-dependent stability criteria are firstly established. Next, state feedback controllers are designed according to the stability conditions in different cases. Finally, five numerical examples are provided to demonstrate the theoretical results.

\section{Introduction}

It is well known that neutral systems are frequently encountered in various engineering systems, including population, ecology, distributed networks containing lossless transmission lines, heat exchangers, and repetitive control. A neutral time-delay system is a special delay system which contains delays both in its state and in its derivatives of state. And the delay in the derivative of state is always called neutral delay, while the delay in the state without derivative is discrete delay. Generally speaking, discrete delay is independent of the neutral delay in neutral delay systems, which means discrete delay is not always equal to neutral delay. In fact, both neutral delay and discrete delay are one kind of time delays in delay systems. In the past two decades, a great deal of attention has been drawn to the delay-dependent stability problem of neutral dynamical systems (see, e.g., [133]). Various important methods have been introduced to obtain the delay-dependent stability conditions for neutral systems, such as model transformation approach [3], delay partitioning technique [4], discretized Lyapunov functional method [6], free-weighting matrix approach [7, 8], and integral inequality method [9]. With this approach, better and better delay-dependent stability conditions are presented one by one, such as $[6,12,17]$ and the references cited therein. Many controller designs are also provided in $[13,34,35]$ and so on. Among those techniques to get stabilization for neutral systems, Jensen's like inequality has played an important role in obtaining delay-dependent stability conditions [10].

However, Jensen's like inequality inevitably introduces an undesirable conservatism and improving it is always an open problem [11]. It has been improved in [11] which dealt with single integral terms. Recently, a new multiple integral inequality was introduced following a similar line as in proof of the Jensen inequality in [12], and a novel delay-dependent stability criterion was established, which has unfortunately observed that the computational burden is slightly heavy. Actually, [13] observed that the upper bounds of double integral terms should also be estimated if triple integral terms are introduced in the Lyapunov-Krasovskii functional to obtain less conservative conditions. Based on the Wirtinger inequality, [14] improved the double Jensen's like double integral, and a double integral form of the Wirtinger-based integral was introduced. However, they did not consider the derivative of the integrand directly. We may get another double integral inequality if we consider this case of derivative. Many articles also attempt to introduce less conservative inequalities [15, 16]. Very recently, [27] presented a new class of inequalities, which produce tighter bounds than what the Jensen inequality produces. These new inequalities will play a key role in the field of stability analysis for 
less conservativeness of delay-dependent stabilization results. This motivates our paper.

In this paper, we investigate the conventional neutral systems to obtain delay-dependent stability and controller design criteria. The rest of the paper is organized as follows. In Section 2, we formulate the problem and the new class of inequalities are provided. In Section 3, the stability and stabilization conditions for neutral systems with mixed delay are presented. The sufficient conditions are formed in terms of LMIs, which can be easily calculated by Matlab control toolbox [25]. Many cases are compared in the tables based on the conventional numerical examples, which are used to show the validity and less conservativeness of our approach in Section 4. The paper is concluded by Section 5 .

\section{Problem Statement and Preliminaries}

In this paper, we will reconsider the stabilization conditions for the following neutral control system with mixed time delays:

$$
\begin{aligned}
\dot{x}(t)-C \dot{x}(t-\tau) & =A x(t)+B x(t-h)+D u(t), \\
x\left(t_{0}+\theta\right) & =\varphi(\theta), \quad \forall \theta \in[-\rho, 0],
\end{aligned}
$$

where $x(t) \in \mathbb{R}^{n}$ is the state vector, $u(t) \in \mathbb{R}^{m}$ is the control input, and $u(t)=K x(t), \tau$, and $h$ are time delays, $\rho=$ $\max \{\tau, h\}$, and $\varphi(\theta)$ is the initial condition function. $A, B, C$ are known matrices and $K$ is unknown matrix to be designed. In this paper, we always assume that the spectral radius of $C$ is less than 1 .

As is well known, inequalities and Lyapunov functionals are both important to improve the delay-dependent stabilization condition for neutral control systems with mixed time delays. Before presenting the main results, we first state the following lemma which is novelty and will be used in the proof of our main results.

Lemma 1 (see [27]). For a positive definite matrix $R>0$ and a differentiable function $\{x(u) \mid u \in[a, b]\}$, the following inequalities hold:

$$
\begin{aligned}
& \int_{a}^{b} x^{T}(\alpha) R x(\alpha) d \alpha \\
& \geq \frac{1}{b-a}\left(\int_{a}^{b} x(\alpha) d \alpha\right)^{T} R\left(\int_{a}^{b} x(\alpha) d \alpha\right) \\
& \quad+\frac{3}{b-a} \Omega_{1}^{T} R \Omega_{1}, \\
& \int_{a}^{b} \dot{x}^{T}(\alpha) R \dot{x}(\alpha) d \alpha \\
& \geq \frac{1}{b-a} \Omega_{2}^{T} R \Omega_{2}+\frac{3}{b-a} \Omega_{3}^{T} R \Omega_{3}+\frac{5}{b-a} \Omega_{4}^{T} R \Omega_{4}, \\
& \int_{a}^{b} \int_{\beta}^{b} \dot{x}^{T}(\alpha) R \dot{x}(\alpha) d \alpha d \beta \geq 2 \Omega_{5}^{T} R \Omega_{5}+4 \Omega_{6}^{T} R \Omega_{6},
\end{aligned}
$$

where

$$
\begin{aligned}
\Omega_{1}= & \int_{a}^{b} x(\alpha) d \alpha-\frac{2}{(b-a)} \int_{a}^{b} \int_{\beta}^{b} x \alpha d \alpha d \beta \\
\Omega_{2}= & x(b)-x(a) \\
\Omega_{3}= & x(b)+x(a)-\frac{2}{b-a} \int_{a}^{b} x(\alpha) d \alpha \\
\Omega_{4}= & x(b)-x(a)+\frac{6}{b-a} \int_{a}^{b} x(\alpha) d \alpha \\
& -\frac{12}{(b-a)^{2}} \int_{a}^{b} \int_{\beta}^{b} x \alpha d \alpha d \beta \\
\Omega_{5}= & x(b)-\frac{1}{b-a} \int_{a}^{b} x(\alpha) d \alpha \\
\Omega_{6}= & x(b)+\frac{2}{b-a} \int_{a}^{b} x(\alpha) d \alpha \\
& -\frac{6}{(b-a)^{2}} \int_{a}^{b} \int_{\beta}^{b} x(\alpha) d \alpha d \beta .
\end{aligned}
$$

Remark 2. In fact, [27] introduced many novelty inequalities. For our purpose for stability analysis, we introduced some of the most important inequalities for our article. One can refer to the other inequalities in [27] and learn the proof of these novelty inequalities, which produce tighter bounds than the Jensen inequality.

Remark 3. Actually, with simple computation, inequality (4) of Lemma 1 can be easily transformed into the form of LMI which can be used thereafter in this paper as follows:

$$
\int_{b}^{a} \int_{\beta}^{b} \dot{x}^{T}(\alpha) R \dot{x}(\alpha) d \alpha d \beta \geq 6 \Omega^{T} \mathscr{R} \Omega,
$$

where

$$
\begin{aligned}
\mathscr{R} & =\left(\begin{array}{ccc}
R & R & -4 R \\
\star & 3 R & -8 R \\
\star & \star & 24 R
\end{array}\right), \\
\Omega^{T} & =\left(x^{T}(t), \frac{1}{b-a} \int_{a}^{b} x^{T}(\alpha) d \alpha, \frac{1}{(b-a)^{2}}\right. \\
& \left.\cdot \int_{a}^{b} \int_{\beta}^{b} x^{T}(\alpha) d \alpha d \beta\right) .
\end{aligned}
$$

\section{Main Result}

In this section, we will firstly introduce the delay-dependent stability conditions for system (1) in different case. By constructing a new class of Lyapunov functionals and using many inequalities which were introduced in Lemma 1, some much better delay-dependent stability conditions are obtained. And, then, based on the obtained delay-dependent stability conditions, the state feedback controllers for system (1) are 
designed by the optimization technique. We are now in a position to derive all the conditions as follows.

Theorem 4. System (1) with $u(t)=0$ is asymptotically stable if there exist positive definite matrices $P, W_{1}, W_{2}, Q_{1}, Q_{2}, R_{1}$, $R_{2}$, symmetric matrices $Y_{i i}, Z_{i i}(i=1,2,3,4), R_{11}, R_{22}$, and any matrices $Y_{i j}, Z_{i j}(i \neq j \in 1,2,3,4), \mathfrak{R}_{2 \times 2}$ with appropriate dimensions satisfying the following linear matrix inequalities:

$$
\begin{array}{r}
\Psi_{6 \times 6}>0, \\
\left(\begin{array}{cc}
\varphi_{8 \times 8} & \Lambda_{1}^{T} \\
\star & -S
\end{array}\right)<0,
\end{array}
$$

where

$$
\begin{aligned}
& \Psi_{11}=P+Y_{11}+Z_{11}+\frac{4}{\tau} R_{22}+6 h W_{2}+6 \tau W_{1}+\frac{3 h}{2} R_{2} \\
& +\frac{3 \tau}{2} R_{1} \\
& \Psi_{12}=Y_{12}+Z_{12}+\frac{2}{\tau} R_{22} \\
& \Psi_{13}=\tau Y_{13}-2 R_{12}^{T}-\frac{6}{\tau} R_{22}+6 \tau W_{1}, \\
& \Psi_{14}=6 h W_{2}+\tau^{2} Z_{13} \text {, } \\
& \Psi_{15}=6 R_{12}^{T}-24 \tau W_{1}-3 \tau R_{1}+h Y_{14}, \\
& \Psi_{16}=-24 h W_{2}-3 h R_{2}+h^{2} Z_{14} \text {, } \\
& \Psi_{22}=Y_{22}+Z_{22}+\frac{4}{\tau} R_{22} \text {, } \\
& \Psi_{23}=\tau Y_{23}-4 R_{12}^{T}-\frac{6}{\tau} R_{22} \text {, } \\
& \Psi_{24}=\tau^{2} Z_{23}, \\
& \Psi_{25}=6 R_{12}^{T}+h Y_{24} \text {, } \\
& \Psi_{26}=h^{2} Z_{24} \text {, } \\
& \Psi_{33}=4 \tau Q_{1}+4 \tau R_{11}+\frac{12}{\tau} R_{22}+6 R_{12}+6 R_{12}^{T}+18 \tau W_{1} \\
& +\tau^{2} Y_{33} \\
& \Psi_{35}=-6 \tau Q_{1}-2 \tau\left(3 R_{11}+\frac{6}{\tau} R_{12}^{T}\right)-48 \tau W_{1}+h \tau Y_{34} \text {, } \\
& \Psi_{44}=4 h Q_{2}+18 h W_{2}+\tau^{4} Z_{33} \text {, } \\
& \Psi_{46}=-6 h Q_{2}-48 h W_{2}+h^{2} \tau^{2} Z_{34}, \\
& \Psi_{55}=12 \tau Q_{1}+12 \tau R_{11}+144 \tau W_{1}+6 \tau R_{1}+h^{2} Y_{44}, \\
& \Psi_{66}=12 h Q_{2}+144 h W_{2}+6 h R_{2}+h^{4} Z_{44} \text {, }
\end{aligned}
$$

$$
\begin{aligned}
& \Lambda_{1}=\left[\begin{array}{llllllll}
S A & 0 & S B & S C & 0 & 0 & 0 & 0
\end{array}\right] \text {, } \\
& \varphi_{11}=Q_{1}+Q_{2}-6 R_{1}+R_{11}-6 R_{2}-9 W_{1}-9 W_{2}+Y_{13} \\
& +Y_{13}^{T}+Y_{14}+Y_{14}^{T}+h Z_{14}+h Z_{14}^{T}+\tau Z_{13} \\
& +\tau Z_{13}^{T}+P A+A^{T} P+Y_{11} A+A^{T} Y_{11}^{T} \\
& +Z_{11} A+A^{T} Z_{11}^{T}+R_{12} A+A^{T} R_{12}^{T}, \\
& \varphi_{12}=Y_{23}^{T}-Y_{13}+3 W_{1}+Y_{24}^{T}+A^{T} Y_{12}+A^{T} Z_{12} \\
& +h Z_{24}^{T}+\tau Z_{23}^{T} \\
& \varphi_{13}=3 W_{2}-Y_{14}+R_{12} B+P B+Y_{11} B+Z_{11} B \text {, } \\
& \varphi_{22}=-Q_{1}-R_{11}-9 W_{1}-Y_{23}-Y_{23}^{T} \text {, } \\
& \varphi_{23}=Y_{12}^{T} B-Y_{24}+Z_{12}^{T} B \text {, } \\
& \varphi_{33}=-9 W_{2}-Q_{2} \text {, } \\
& \varphi_{14}=Y_{12}+Z_{12}+R_{12} C+P C+Y_{11} C+Z_{11} C, \\
& \varphi_{24}=Y_{22}-R_{12}+Z_{22}+Y_{12}^{T} C+Z_{12}^{T} C \text {, } \\
& \varphi_{44}=-R_{22} \text {, } \\
& \varphi_{15}=\tau Y_{33}+\tau Y_{34}^{T}+\tau A^{T} Y_{13}-24 W_{1}-\tau Z_{13}-6 R_{1}, \\
& \varphi_{25}=36 W_{1}-\tau Y_{33}-\tau Z_{23} \text {, } \\
& \varphi_{35}=-\tau\left(Y_{34}^{T}-B^{T} Y_{13}\right) \text {, } \\
& \varphi_{45}=\tau\left(Y_{23}+C^{T} Y_{13}\right) \text {, } \\
& \varphi_{55}=-18 R_{1}-192 W_{1} \text {, } \\
& \varphi_{16}=h\left(Y_{34}+Y_{44}+A^{T} Y_{14}\right)-24 W_{2}-h Z_{14}-6 R_{2} \text {, } \\
& \varphi_{26}=-h Y_{34}-h Z_{24} \text {, } \\
& \varphi_{36}=36 W_{2}-h\left(Y_{44}-B^{T} Y_{14}\right), \\
& \varphi_{46}=h\left(Y_{24}+C^{T} Y_{14}\right) \text {, } \\
& \varphi_{66}=-18 R_{2}-192 W_{2} \text {, } \\
& \varphi_{17}=24 R_{1}+60 W_{1}+\tau^{2}\left(A^{T} Z_{13}+\tau Z_{33}+h Z_{34}^{T}\right) \text {, } \\
& \varphi_{27}=-60 W_{1} \text {, } \\
& \varphi_{37}=\tau^{2} B^{T} Z_{13} \\
& \varphi_{47}=\tau^{2}\left(Z_{23}+C^{T} Z_{13}\right), \\
& \varphi_{57}=48 R_{1}+360 W_{1}-\tau^{3} Z_{33}, \\
& \varphi_{67}=-h \tau^{2} Z_{34}^{T} \text {, } \\
& \varphi_{77}=-144 R_{1}-720 W_{1} \text {, } \\
& \varphi_{18}=24 R_{2}+60 W_{2}+h^{2}\left(A^{T} Z_{14}+h Z_{44}+\tau Z_{34}\right) \text {, }
\end{aligned}
$$




$$
\begin{aligned}
\varphi_{38} & =h^{2} B^{T} Z_{14}-60 W_{2}, \\
\varphi_{48} & =h^{2}\left(Z_{24}+C^{T} Z_{14}\right), \\
\varphi_{58} & =-\tau h^{2} Z_{34}, \\
\varphi_{68} & =48 R_{2}+360 W_{2}-h^{3} Z_{44}, \\
\varphi_{88} & =-144 R_{2}-720 W_{2}, \\
S & =R_{22}+\frac{\tau^{2}}{2} R_{1}+\frac{h^{2}}{2} R_{2}+\tau^{2} W_{1}+h^{2} W_{2} .
\end{aligned}
$$

Proof. Construct a Lyapunov functional candidate as

$$
V\left(x_{t}, t\right)=\sum_{i=1}^{5} V_{i}\left(x_{t}, t\right),
$$

with

$$
\begin{aligned}
V_{1}= & x^{T}(t) P x(t) \\
V_{2}= & \int_{t-\tau}^{t} x^{T}(s) Q_{1} x(s) d s+\int_{t-h}^{t} x^{T}(s) Q_{2} x(s) d s \\
& +\int_{t-\tau}^{t}\left(\begin{array}{c}
x(s) \\
\dot{x}(s)
\end{array}\right)^{T} \Re\left(\begin{array}{c}
x(s) \\
\dot{x}(s)
\end{array}\right) d s \\
V_{3}= & \int_{t-\tau}^{t} \int_{\beta}^{t} \dot{x}^{T}(\alpha)\left(\tau W_{1}\right) \dot{x}(\alpha) d \alpha d \beta \\
& +\int_{t-h}^{t} \int_{\beta}^{t} \dot{x}^{T}(\alpha)\left(h W_{2}\right) \dot{x}(\alpha) d \alpha d \beta, \\
V_{4}= & \int_{t-\tau}^{t} \int_{\theta}^{t} \int_{\lambda}^{t} \dot{x}^{T}(s) R_{1} \dot{x}(s) d s d \lambda d \theta \\
& +\int_{t-h}^{t} \int_{\theta}^{t} \int_{\lambda}^{t} \dot{x}^{T}(s) R_{2} \dot{x}(s) d s d \lambda d \theta, \\
V_{5}= & G_{1}^{T} Z G_{1}+G_{2}^{T} Y G_{2},
\end{aligned}
$$

where $Y$ and $Z$ are 4-order matrices with their elements $Y_{i j}$, $Z_{i j}(i, j=1,2,3,4)$ expressed in Theorem 4 .

$$
\begin{aligned}
G_{1} & =\left(\begin{array}{c}
x(t) \\
x(t-\tau) \\
\int_{t-\tau}^{t} \int_{\theta}^{t} x(s) d s d \theta \\
\int_{t-h}^{t} \int_{\theta}^{t} x(s) d s d \theta
\end{array}\right) \\
G_{2}= & \left(\begin{array}{c}
x(t) \\
x(t-\tau) \\
\int_{t-\tau}^{t} x(s) d s \\
\int_{t-h}^{t} x(s) d s
\end{array}\right)
\end{aligned}
$$

For the sake of convenience, we set $e_{i} \in \mathbb{R}^{8 n \times n}(i=$ $1, \ldots, 8)$ as elementary matrices; for example, $e_{2}^{T}=$ $\left[\begin{array}{llllllll}0 & I & 0 & 0 & 0 & 0 & 0 & 0\end{array}\right]$, and let

$$
\begin{aligned}
e_{0} & =A e_{1}+B e_{3}+C e_{4}, \\
\xi^{T} & =\left(x^{T}(t), x^{T}(t-\tau), x^{T}(t-h), \dot{x}^{T}(t-\tau), \frac{1}{\tau}\right. \\
& \cdot \int_{t-\tau}^{t} x^{T}(s) d s, \frac{1}{h} \int_{t-h}^{t} x^{T}(s) d s, \frac{1}{\tau^{2}} \\
& \left.\cdot \int_{t-\tau}^{t} \int_{\theta}^{t} x^{T}(s) d s d \theta, \frac{1}{h^{2}} \int_{t-h}^{t} \int_{\theta}^{t} x^{T}(s) d s d \theta\right) .
\end{aligned}
$$

First, with Lemma 1, the Lyapunov functionals $V_{2}$ and $V_{3}$ can be scaled as

$$
\begin{aligned}
V_{2} & \geq \xi^{T} \times\left\{\tau e_{5}^{T} Q_{1} e_{5}+3 \tau\left(e_{5}-2 e_{7}\right)^{T} Q_{1}\left(e_{5}-2 e_{7}\right)\right. \\
& +h e_{6}^{T} Q_{2} e_{6}+3 h\left(e_{6}-2 e_{8}\right)^{T} Q_{2}\left(e_{6}-2 e_{8}\right) \\
& +\frac{1}{\tau}\left[\tau e_{5}^{T} e_{1}^{T}-e_{2}^{T}\right] \Re\left[\tau e_{5}^{T} e_{1}^{T}-e_{2}^{T}\right]^{T} \\
& +\frac{3}{\tau}\left[\tau e_{5}^{T}-2 \tau e_{7}^{T}-e_{1}^{T}-e_{2}^{T}+2 e_{5}^{T}\right] \Re \\
& \left.\times\left[\tau e_{5}^{T}-2 \tau e_{7}^{T}-e_{1}^{T}-e_{2}^{T}+2 e_{5}^{T}\right]^{T}\right\} \times \xi \\
V_{3} & \geq \xi^{T} \times\left\{2 \tau\left(e_{1}-e_{5}\right)^{T} W_{1}\left(e_{1}-e_{5}\right)\right. \\
& +4 \tau\left(e_{1}+2 e_{5}-6 e_{7}\right)^{T} W_{1}\left(e_{1}+2 e_{5}-6 e_{7}\right) \\
& +2 h\left(e_{1}-e_{6}\right)^{T} W_{2}\left(e_{1}-e_{6}\right) \\
& \left.+4 h\left(e_{1}+2 e_{6}-6 e_{8}\right)^{T} W_{2}\left(e_{1}+2 e_{6}-6 e_{8}\right)\right\} \xi .
\end{aligned}
$$

Following the same line to prove the conventional Jensen inequality [9], $V_{4}$ can be bounded as

$$
\begin{aligned}
V_{4} & \geq \xi^{T} \times\left\{\frac{6}{\tau^{3}}\left(\frac{\tau^{2}}{2} e_{1}-\tau^{2} e_{7}\right)^{T} R_{1}\left(\frac{\tau^{2}}{2} e_{1}-\tau^{2} e_{7}\right)\right. \\
& \left.+\frac{6}{h^{3}}\left(\frac{h^{2}}{2} e_{1}-h^{2} e_{8}\right)^{T} R_{2}\left(\frac{h^{2}}{2} e_{1}-h^{2} e_{8}\right)\right\} \xi .
\end{aligned}
$$

On the other hand, $V_{1}$ and $V_{5}$ can be presented as

$$
\begin{aligned}
V_{1} & =\xi^{T} e_{1}^{T} P e_{1} \xi \\
V_{5} & =\xi^{T} \times\left\{\left[\begin{array}{llll}
e_{1}^{T} & e_{2}^{T} & \tau^{2} e_{7}^{T} & h^{2} e_{8}^{T}
\end{array}\right]^{T} \times Z\right. \\
& \times\left[\begin{array}{llll}
e_{1}^{T} & e_{2}^{T} & \tau^{2} e_{7}^{T} & h^{2} e_{8}^{T}
\end{array}\right] \\
& \left.+\left[\begin{array}{llll}
e_{1}^{T} & e_{2}^{T} & \tau e_{5}^{T} & h e_{6}^{T}
\end{array}\right]^{T} Y\left[\begin{array}{llll}
e_{1}^{T} & e_{2}^{T} & \tau e_{5}^{T} & h e_{6}^{T}
\end{array}\right]\right\} \xi .
\end{aligned}
$$

From (15) to (17), we can get that

$$
V \geq \xi_{1}^{T} \Psi \xi_{1}
$$

where 


$$
\xi_{1}^{T}=\left(x^{T}(t), x^{T}(t-\tau), \frac{1}{\tau} \int_{t-\tau}^{t} x^{T}(s) d s, \frac{1}{h} \int_{t-h}^{t} x^{T}(s) d s, \frac{1}{\tau^{2}} \int_{t-\tau}^{t} \int_{\theta}^{t} x^{T}(s) d s d \theta, \frac{1}{h^{2}} \int_{t-h}^{t} \int_{\theta}^{t} x^{T}(s) d s d \theta\right)
$$

According to inequality (8), functional (11) is positive definite. Then, the time derivative of $V(t)$ along the trajectories of (1) can be firstly computed as follows:

$$
\dot{V}\left(x_{t}, t\right)=\sum_{i=1}^{5} \dot{V}_{i}\left(x_{t}, t\right)
$$

with

$$
\begin{aligned}
& \dot{V}_{1}=2 x^{T}(t) P[A x(t)+B x(t-h)+C \dot{x}(t-h)] \\
& =2 \xi^{T} e_{1}^{T} P e_{0} \xi \\
& \dot{V}_{2}=x^{T}(t)\left(Q_{1}+Q_{2}\right) x(t)-x^{T}(t-\tau) Q_{1} x(t-\tau) \\
& -x^{T}(t-h) Q_{2} x(t-h)+\left(\begin{array}{c}
x(t) \\
\dot{x}(t)
\end{array}\right)^{T} \mathfrak{R}\left(\begin{array}{c}
x(t) \\
\dot{x}(t)
\end{array}\right) \\
& -\left(\begin{array}{c}
x(t-\tau) \\
\dot{x}(t-\tau)
\end{array}\right)^{T} \mathfrak{R}\left(\begin{array}{c}
x(t-\tau) \\
\dot{x}(t-\tau)
\end{array}\right)^{T} \\
& =\xi^{T}\left[\left(\begin{array}{l}
e_{1} \\
e_{0}
\end{array}\right)^{T} \mathfrak{R}\left(\begin{array}{l}
e_{1} \\
e_{0}
\end{array}\right)-\left(\begin{array}{l}
e_{2} \\
e_{4}
\end{array}\right)^{T} \mathfrak{R}\left(\begin{array}{l}
e_{2} \\
e_{4}
\end{array}\right)\right. \\
& \left.+e_{1}^{T}\left(Q_{1}+Q_{2}\right) e_{1}-e_{2}^{T} Q_{1} e_{2}-e_{3}^{T} Q_{2} e_{3}\right] \xi, \\
& \dot{V}_{3}=\xi^{T}\left[e_{0}^{T}\left(\tau^{2} W_{1}+h^{2} W_{2}\right) e_{0}\right] \xi \\
& -\tau \int_{t-\tau}^{t} \dot{x}^{T}(s) W_{1} \dot{x}(s) d s \\
& -h \int_{t-h}^{t} \dot{x}^{T}(s) W_{2} \dot{x}(s) d s .
\end{aligned}
$$

With Lemma 1, one can obtain that

$$
\begin{aligned}
\dot{V}_{3} & \leq \xi^{T}\left[e_{0}^{T}\left(\tau^{2} W_{1}+h^{2} W_{2}\right) e_{0}-\left(e_{1}-e_{2}\right)^{T}\right. \\
& \cdot W_{1}\left(e_{1}-e_{2}\right)-\left(e_{1}-e_{3}\right)^{T} W_{2}\left(e_{1}-e_{3}\right) \\
& -3\left(e_{1}+e_{2}-2 e_{5}\right)^{T} W_{1}\left(e_{1}+e_{2}-2 e_{5}\right) \\
& -3\left(e_{1}+e_{3}-2 e_{6}\right)^{T} W_{2}\left(e_{1}+e_{3}-2 e_{6}\right) \\
& -5\left(e_{1}-e_{2}+6 e_{5}-12 e_{7}\right)^{T} \\
& \cdot W_{1}\left(e_{1}-e_{2}+6 e_{5}-12 e_{7}\right)
\end{aligned}
$$

$$
\begin{gathered}
-5\left(e_{1}-e_{3}+6 e_{6}-12 e_{8}\right)^{T} \\
\left.\cdot W_{2}\left(e_{1}-e_{3}+6 e_{6}-12 e_{8}\right)\right] \xi, \\
\dot{V}_{4}=\xi^{T}\left[\frac{1}{2} e_{0}^{T}\left(\tau^{2} R_{1}+h^{2} R_{2}\right) e_{0}\right] \xi \\
-\int_{t-\tau}^{t} \int_{\theta}^{t} \dot{x}^{T}(s) R_{1} x(s) d s d \theta \\
-\int_{t-h}^{t} \int_{\theta}^{t} \dot{x}^{T}(s) R_{2} x(s) d s d \theta .
\end{gathered}
$$

By using inequality (6) for Lemma 1, one can see that

$$
\begin{aligned}
\dot{V}_{4} & \leq \xi^{T}\left[\frac{1}{2} e_{0}^{T}\left(\tau^{2} R_{1}+h^{2} R_{2}\right) e_{0}\right. \\
& -\left(\begin{array}{lll}
e_{1}^{T} & e_{5}^{T} & e_{7}^{T}
\end{array}\right) K_{1}\left(\begin{array}{lll}
e_{1}^{T} & e_{5}^{T} & e_{7}^{T}
\end{array}\right)^{T} \\
& \left.-\left(\begin{array}{lll}
e_{1}^{T} & e_{6}^{T} & e_{8}^{T}
\end{array}\right) K_{2}\left(\begin{array}{lll}
e_{1}^{T} & e_{6}^{T} & e_{8}^{T}
\end{array}\right)^{T}\right] \xi,
\end{aligned}
$$

with

$$
\begin{aligned}
& K_{1}=6 R_{1}\left(\begin{array}{ccc}
1 & 1 & -4 \\
1 & 3 & -8 \\
-4 & -8 & 24
\end{array}\right) \text {, } \\
& K_{2}=6 R_{2}\left(\begin{array}{ccc}
1 & 1 & -4 \\
1 & 3 & -8 \\
-4 & -8 & 24
\end{array}\right) \text {, } \\
& \dot{V}_{5}=\dot{G}_{1}^{T} Z G_{1}+G_{1}^{T} Z \dot{G}_{1}+\dot{G}_{2}^{T} Y G_{2}+G_{2}^{T} Y \dot{G}_{2} \\
& =\xi^{T}\left[\begin{array}{lllll}
2\left(e_{1}^{T}\right. & e_{2}^{T} & \tau^{2} e_{7}^{T} & h^{2} e_{8}^{T}
\end{array}\right)^{T} \\
& \cdot Z\left(\begin{array}{llll}
e_{0}^{T} & e_{4}^{T} & \tau e_{1}^{T}-\tau e_{5}^{T} h e_{1}^{T}-h e_{6}^{T}
\end{array}\right)^{T} \\
& \left.+2\left(\begin{array}{llll}
e_{1}^{T} & e_{2}^{T} & \tau e_{5}^{T} & h e_{6}^{T}
\end{array}\right) Y\left(\begin{array}{llll}
e_{0}^{T} & e_{4}^{T} & e_{1}^{T}-e_{2}^{T} & e_{1}^{T}-e_{3}^{T}
\end{array}\right)^{T}\right] \\
& \text {. } \xi
\end{aligned}
$$

From (20) to (24), one can see that

$$
\dot{V}=\xi^{T}\left(\varphi+\Lambda_{1}^{T} S^{-1} \Lambda_{1}\right) \xi<0 .
$$

Using Schur's complement lemma, it is obvious that inequality (25) holds if condition (9) is satisfied in Theorem 4. Accordingly, system (1) is asymptotically stable, which concludes the proof. 
Remark 5. In the proof of this theorem, functional $V$ is more general than the existing results for considering much more information about the state vector. In particular, $V_{5}$ is very important to improve the delay-dependent stability condition according to many numerical experiments. First, the vector of the augmented Lyapunov functional should contain $x(t-\tau)$. In other words, if one attempts to delete $x(t-\tau)$ from $G_{1}$ or $G_{2}$, the maximum of time delay may be less than those of the original $V_{5}$. Second, single integral of $\tau$ and $h$ should be presented in $G_{1}$ at the same time; it is similar to double integral in $G_{2}$. More accurately, single integral and double integral should not be put together in $G_{1}$ or $G_{2}$. It is also very important to use a new class of inequalities for improving delay-dependent stability condition.

Remark 6. The considered neutral systems with constant delays have been well studied so far with very rich literature. However, we would obtain the best condition comapred to the existing results based on the skillful combination of our novelty constructed Lyapunov functionals and the new class of inequalities.

When the neutral delay $\tau$ is equal to discrete delay $h$, following the same line of the proof for Theorem 4, we can obtain the following theorem.

Theorem 7. System (1) with $u(t)=0$ and $\tau=h$ is asymptotically stable if there exist positive definite matrices $P, W_{2}, Q_{2}, R_{2}$, symmetric matrices $Y_{i i}, Z_{i i}(i=1,2,3), R_{11}$, $R_{22}$, and any matrices $Y_{i j}, Z_{i j}(i \neq j \in 1,2,3), \mathfrak{R}_{2 \times 2}$ with appropriate dimensions satisfying the following linear matrix inequalities:

$$
\begin{array}{r}
\Psi_{4 \times 4}>0 \\
\left(\begin{array}{cc}
\varphi_{5 \times 5} & \Lambda_{2}^{T} \\
\star & -S
\end{array}\right)<0
\end{array}
$$

where

$$
\begin{aligned}
& \Psi_{11}=P+Y_{11}+Z_{11}+6 h W_{2}+\frac{4}{h} R_{22}+\frac{3 h}{2} R_{2}, \\
& \Psi_{12}=Y_{12}+Z_{12}+\frac{2}{h} R_{22}, \\
& \Psi_{13}=6 h W_{2}-2 R_{12}^{T}+h Y_{13}-\frac{6}{h} R_{22}, \\
& \Psi_{14}=6 R_{12}^{T}-24 h W_{2}+h^{2} Z_{13}-\frac{3 h}{2} R_{2}, \\
& \Psi_{22}=Y_{22}+Z_{22}+\frac{4}{h} R_{22} \\
& \Psi_{23}=h Y_{23}-4 R_{12}^{T}-\frac{6}{h} R_{22}, \\
& \Psi_{24}=h^{2} Z_{23}+6 R_{12}^{T},
\end{aligned}
$$

$$
\begin{aligned}
& \Psi_{33}=4 h Q_{2}+18 h W_{2}+6 R_{12}+6 R_{12}^{T}+4 h R_{11} \\
& +\frac{12}{h} R_{22}+h^{2} Y_{33} \\
& \Psi_{34}=-6 h Q_{2}-48 h W_{2}-12 R_{12}^{T}-6 h R_{11} \text {, } \\
& \Psi_{44}=12 h Q_{2}+144 h W_{2}+12 h R_{11}+6 h R_{2}+h^{4} Z_{33} \text {, } \\
& \Lambda_{2}=\left[\begin{array}{llllll}
S A & S B & S C & 0 & 0 & 0
\end{array}\right], \\
& \varphi_{11}=R_{11}-6 R_{2}-9 W_{2}+Y_{13}+Y_{13}^{T}+h Z_{13}+h Z_{13}^{T} \\
& +A^{T} P+P A+A^{T} Y_{11}+Y_{11} A+Z_{11} A \\
& +A^{T} Z_{11}+Q_{2}+R_{12} A+A^{T} R_{12}^{T} \text {, } \\
& \varphi_{12}=3 W_{2}-Y_{13}+Y_{23}^{T}+A^{T} Y_{12}+A^{T} Z_{12}+h Z_{23}^{T} \\
& +\left(P+Y_{11}+Z_{11}+R_{12}\right) B, \\
& \varphi_{13}=Y_{12}+Z_{12}+R_{12} C+P C+Y_{11} C+Z_{11} C \text {, } \\
& \varphi_{14}=h\left(Y_{33}+A^{T} Y_{13}\right)-24 W_{2}-h Z_{13}-6 R_{2} \text {, } \\
& \varphi_{15}=24 R_{2}+60 W_{2}+h^{2}\left(A^{T} Z_{13}+h Z_{33}\right), \\
& \varphi_{22}=B^{T} Y_{12}+Y_{12}^{T} B-R_{11}-9 W_{2}-Y_{23}-Y_{23}^{T}-Q_{2} \\
& +B^{T} Z_{12}+Z_{12}^{T} B \\
& \varphi_{23}=Y_{22}-R_{12}+Z_{22}+Y_{12}^{T} C+Z_{12}^{T} C \text {, } \\
& \varphi_{24}=36 W_{2}-h Z_{23}-h\left(Y_{33}-B^{T} Y_{13}\right) \text {, } \\
& \varphi_{25}=h^{2} B^{T} Z_{13}-60 W_{2} \text {, } \\
& \varphi_{33}=-R_{22} \text {, } \\
& \varphi_{34}=h\left(Y_{23}+C^{T} Y_{13}\right) \text {, } \\
& \varphi_{35}=h^{2}\left(Z_{23}+C^{T} Z_{13}\right) \text {, } \\
& \varphi_{44}=-18 R_{2}-192 W_{2} \text {, } \\
& \varphi_{45}=48 R_{2}+360 W_{2}-h^{3} Z_{33} \text {, } \\
& \varphi_{55}=-144 R_{2}-720 W_{2} \text {, } \\
& S=R_{22}+\frac{h^{2}}{2} R_{2}+h^{2} W_{2} .
\end{aligned}
$$

Next, based on the above theorems, we will design the input controller $K$ for the neutral control system (1) in different cases.

Theorem 8. System (1) is asymptotically stabilized if there exist positive definite matrices $X, \widehat{W}_{1}, \widehat{W}_{2}, \widehat{Q}_{1}, \widehat{Q}_{2}, \widehat{R}_{1}, \widehat{R}_{2}$, symmetric matrices $\widehat{Y}_{i i}, \widehat{Z}_{i i}(i=1,2,3,4), \widehat{R}_{11}, \widehat{R}_{22}$, and any matrices 
$\widehat{Y}_{i j}, \widehat{Z}_{i j}(i \neq j \in 1,2,3,4), \widehat{R}_{12}$, L with appropriate dimensions satisfying the following linear matrix inequalities:

$$
\widehat{\Psi}_{6 \times 6}>0,
$$

$$
\left(\begin{array}{cccc}
\widehat{\varphi}_{8 \times 8} & 0 & \Lambda_{3}^{T} & \Gamma_{1}^{T} \\
\star & -\widehat{S} & \widehat{S} & 0 \\
\star & \star & -J & 0 \\
\star & \star & \star & -X J^{-1} X
\end{array}\right)<0,
$$

where

$$
\begin{aligned}
\widehat{\Psi}_{11} & \\
= & +\widehat{Y}_{11}+\widehat{Z}_{11}+\frac{4}{\tau} \widehat{R}_{22}+6 h \widehat{W}_{2}+6 \tau \widehat{W}_{1}+\frac{3 h}{2} \widehat{R}_{2} \\
& +\frac{3 \tau}{2} \widehat{R}_{1}, \\
\widehat{\Psi}_{12} & =\widehat{Y}_{12}+\widehat{Z}_{12}+\frac{2}{\tau} \widehat{R}_{22}, \\
\widehat{\Psi}_{13} & =\tau \widehat{Y}_{13}-2 \widehat{R}_{12}^{T}-\frac{6}{\tau} \widehat{R}_{22}+6 \tau \widehat{W}_{1}, \\
\widehat{\Psi}_{14} & =6 h \widehat{W}_{2}+\tau^{2} \widehat{Z}_{13}, \\
\widehat{\Psi}_{15} & =6 \widehat{R}_{12}^{T}-24 \tau \widehat{W}_{1}-3 \tau \widehat{R}_{1}+h \widehat{Y}_{14}, \\
\widehat{\Psi}_{16} & =-24 h \widehat{W}_{2}-3 h \widehat{R}_{2}+h^{2} \widehat{Z}_{14}, \\
\widehat{\Psi}_{22} & =\widehat{Y}_{22}+\widehat{Z}_{22}+\frac{4}{\tau} \widehat{R}_{22}, \\
\widehat{\Psi}_{23} & =\tau \widehat{Y}_{23}-4 \widehat{R}_{12}^{T}-\frac{6}{\tau} \widehat{R}_{22}, \\
\widehat{\Psi}_{24} & =\tau^{2} \widehat{Z}_{23}, \\
\widehat{\Psi}_{25} & =6 \widehat{R}_{12}^{T}+h \widehat{Y}_{24}, \\
\widehat{\Psi}_{26} & =h^{2} \widehat{Z}_{24}, \\
\widehat{\Psi}_{33} & +\tau \widehat{Y}_{33}, \\
= & \tau \widehat{Q}_{1}+4 \tau \widehat{R}_{11}+\frac{12}{\tau} \widehat{R}_{22}+6 \widehat{R}_{12}+6 \widehat{R}_{12}^{T}+18 \tau \widehat{W}_{1}
\end{aligned}
$$$$
\widehat{\Psi}_{35}=-6 \tau \widehat{Q}_{1}-2 \tau\left(3 \widehat{R}_{11}+\frac{6}{\tau} \widehat{R}_{12}^{T}\right)-48 \tau \widehat{W}_{1}+h \tau \widehat{Y}_{34},
$$$$
\widehat{\Psi}_{44}=4 h \widehat{Q}_{2}+18 h \widehat{W}_{2}+\tau^{4} \widehat{Z}_{33} \text {, }
$$$$
\widehat{\Psi}_{46}=-6 h \widehat{Q}_{2}-48 h \widehat{W}_{2}+h^{2} \tau^{2} \widehat{Z}_{34} \text {, }
$$$$
\widehat{\Psi}_{55}=12 \tau \widehat{Q}_{1}+12 \tau \widehat{R}_{11}+144 \tau \widehat{W}_{1}+6 \tau \widehat{R}_{1}+h^{2} \widehat{Y}_{44},
$$

$\widehat{\Psi}_{66}=12 h \widehat{Q}_{2}+144 h \widehat{W}_{2}+6 h \widehat{R}_{2}+h^{4} \widehat{Z}_{44}$,

$\Lambda_{3}$

$=\left[\begin{array}{lllllllll}\widehat{Y}_{11}+\widehat{Z}_{11}+\widehat{R}_{12}^{T} & \widehat{Y}_{12}+\widehat{Z}_{12} & 0 & 0 & \widehat{Y}_{13} & \widehat{Y}_{14} & \widehat{Z}_{13} & \widehat{Z}_{14}\end{array}\right]$,

$\Gamma_{1}=\left[\begin{array}{llllllll}A X+D L & 0 & B X & C X & 0 & 0 & 0 & 0\end{array}\right]$,

$\widehat{\varphi}_{11}$

$$
\begin{aligned}
= & \widehat{Q}_{1}+\widehat{Q}_{2}-6 \widehat{R}_{1}+\widehat{R}_{11}-6 \widehat{R}_{2}-9 \widehat{W}_{1}-9 \widehat{W}_{2}+\widehat{Y}_{13}+\widehat{Y}_{13}^{T} \\
& +\widehat{Y}_{14}+\widehat{Y}_{14}^{T}+h \widehat{Z}_{14}+h \widehat{Z}_{14}^{T}+\tau \widehat{Z}_{13}+\tau \widehat{Z}_{13}^{T}+A X \\
& +X A^{T}+D L+L^{T} D^{T},
\end{aligned}
$$

$\widehat{\varphi}_{12}=\widehat{Y}_{23}^{T}-\widehat{Y}_{13}+3 \widehat{W}_{1}+\widehat{Y}_{24}^{T}+h \widehat{Z}_{24}^{T}+\tau \widehat{Z}_{23}^{T}$,

$\widehat{\varphi}_{22}=-\widehat{Q}_{1}-\widehat{R}_{11}-9 \widehat{W}_{1}-\widehat{Y}_{23}-\widehat{Y}_{23}^{T}$,

$\widehat{\varphi}_{13}=3 \widehat{W}_{2}-\widehat{Y}_{14}+B X$,

$\widehat{\varphi}_{23}=-\widehat{Y}_{24}$,

$\widehat{\varphi}_{33}=-9 \widehat{W}_{2}-\widehat{Q}_{2}$,

$\widehat{\varphi}_{14}=\widehat{Y}_{12}+\widehat{Z}_{12}+C X$,

$\widehat{\varphi}_{24}=\widehat{Y}_{22}-\widehat{R}_{12}+\widehat{Z}_{22}$,

$\widehat{\varphi}_{44}=-\widehat{R}_{22}$,

$\widehat{\varphi}_{15}=\tau \widehat{Y}_{33}+\tau \widehat{Y}_{34}^{T}-24 \widehat{W}_{1}-\tau \widehat{Z}_{13}-6 \widehat{R}_{1}$,

$\widehat{\varphi}_{25}=36 \widehat{W}_{1}-\tau \widehat{Y}_{33}-\tau \widehat{Z}_{23}$,

$\widehat{\varphi}_{35}=-\tau \widehat{Y}_{34}^{T}$,

$\widehat{\varphi}_{45}=\tau \widehat{Y}_{23}$,

$\widehat{\varphi}_{55}=-18 \widehat{R}_{1}-192 \widehat{W}_{1}$,

$\widehat{\varphi}_{16}=h\left(\widehat{Y}_{34}+\widehat{Y}_{44}\right)-24 \widehat{W}_{2}-h \widehat{Z}_{14}-6 \widehat{R}_{2}$,

$\widehat{\varphi}_{26}=-h \widehat{Y}_{34}-h \widehat{Z}_{24}$,

$\widehat{\varphi}_{36}=36 \widehat{W}_{2}-h \widehat{Y}_{44}$,

$\widehat{\varphi}_{46}=h \widehat{Y}_{24}$,

$\widehat{\varphi}_{66}=-18 \widehat{R}_{2}-192 \widehat{W}_{2}$,

$\widehat{\varphi}_{17}=24 \widehat{R}_{1}+60 \widehat{W}_{1}+\tau^{2}\left(\tau \widehat{Z}_{33}+h \widehat{Z}_{34}^{T}\right)$,

$\widehat{\varphi}_{27}=-60 \widehat{W}_{1}$,

$\widehat{\varphi}_{47}=\tau^{2} \widehat{Z}_{23}$,

$\widehat{\varphi}_{57}=48 \widehat{R}_{1}+360 \widehat{W}_{1}-\tau^{3} \widehat{Z}_{33}$,

$\widehat{\varphi}_{67}=-h \tau^{2} \widehat{Z}_{34}^{T}$

$\widehat{\varphi}_{77}=-144 \widehat{R}_{1}-720 \widehat{W}_{1}$,

$\widehat{\varphi}_{18}=24 \widehat{R}_{2}+60 \widehat{W}_{2}+h^{2}\left(h \widehat{Z}_{44}+\tau \widehat{Z}_{34}\right)$, 


$$
\begin{aligned}
& \widehat{\varphi}_{38}=-60 \widehat{W}_{2}, \\
& \widehat{\varphi}_{48}=h^{2} \widehat{Z}_{24}, \\
& \widehat{\varphi}_{58}=-\tau h^{2} \widehat{Z}_{34}, \\
& \widehat{\varphi}_{68}=48 \widehat{R}_{2}+360 \widehat{W}_{2}-h^{3} \widehat{Z}_{44}, \\
& \widehat{\varphi}_{88}=-144 \widehat{R}_{2}-720 \widehat{W}_{2}, \\
& \widehat{S}=\widehat{R}_{22}+\frac{\tau^{2}}{2} \widehat{R}_{1}+\frac{h^{2}}{2} \widehat{R}_{2}+\tau^{2} \widehat{W}_{1}+h^{2} \widehat{W}_{2},
\end{aligned}
$$

and the state feedback controller is $u(t)=L X^{-1} x(t)$.

Proof. By introducing $u(t)=K x(t)$ into system (1), one can obtain that

$$
\dot{x}(t)-C \dot{x}(t-\tau)=(A+D K) x(t)+B x(t-h),
$$

and we replace the element $\Lambda$ in LMI (9) with $A+D K$. Now, pre- and postmultiply $\operatorname{diag}\left\{P^{-1}, P^{-1}, P^{-1}, P^{-1}, P^{-1}, P^{-1}\right\}$ and $\operatorname{diag}\left\{P^{-1}, P^{-1}, P^{-1}, P^{-1}, P^{-1}, P^{-1}, P^{-1}, P^{-1}, P^{-1}\right\}$ to $(8)$ and (9), respectively, and apply the change of variables such that $X \triangleq P^{-1}, \widehat{(*)} \triangleq X \cdot(*) \cdot X$, and $L \triangleq K P^{-1}$. Inequality (28) can be easily obtained. On the other hand, we obtain

$$
\Xi+\Lambda_{3}^{T} X^{-1} \Gamma_{1}+\Gamma_{1}^{T} X^{-1} \Lambda_{3}<0,
$$

where

$$
\begin{aligned}
& \Xi=\left(\begin{array}{cc}
\widehat{\varphi}_{8 \times 8} & 0 \\
\star & \widehat{S}
\end{array}\right) \\
& \Lambda_{3} \\
& =\left[\begin{array}{lllllllll}
\widehat{Y}_{11}+\widehat{Z}_{11}+\widehat{R}_{12}^{T} & \widehat{Y}_{12}+\widehat{Z}_{12} & 0 & 0 & \widehat{Y}_{13} & \widehat{Y}_{14} & \widehat{Z}_{13} & \widehat{Z}_{14}
\end{array}\right], \\
& \Gamma_{1}=\left[\begin{array}{llllllll}
A X+D L & 0 & B X & C X & 0 & 0 & 0 & 0
\end{array}\right] .
\end{aligned}
$$

Clearly, the following inequality is satisfied for any $J>0$ with appropriate dimensions:

$$
\Lambda_{3}^{T} X^{-1} \Gamma_{1}+\Gamma_{1}^{T} X^{-1} \Lambda_{3} \leq \Lambda_{3}^{T} J^{-1} \Lambda_{3}+\Gamma_{1}^{T} X^{-1} J X^{-1} \Gamma_{1} .
$$

By introducing the above inequality (35) into (32) and using Schur's complement lemma, inequality (29) is obtained easily. This completes the proof.

It is noted that the resulting conditions for the controller design in Theorem 8 are no longer LMI conditions because of the term $X J^{-1} X$ in (29). As a result, unfortunately, we cannot find in general the global maximum time delay using convex optimization algorithms in this case to design controller.

An easy way to obtain a suboptimal maximal delay instead is simply setting $J=\lambda X$ in (28) and (29), which results in LMI conditions. However, this approach will bring much conservativeness. If one can afford more computational efforts, better results can be obtained using an iterative algorithm presented next, which is usually adopted in many references such as [29].
First, we define a new variable $N>0$ such that $X^{-1} J X^{-1} \leq$ $N^{-1}$. Let $H=N^{-1}, M=X^{-1}$, and $F=J^{-1}$; computing the feasible solution of (29) can be transformed to resolve the minimization problem of the following LMI constraints:

$$
\begin{aligned}
\min \quad & \operatorname{Trace}(N H+X M+J F) \\
\text { s.t. } & \left(\begin{array}{cc}
H & M \\
M & F
\end{array}\right) \geq 0, \\
& \left(\begin{array}{ll}
N & I \\
I & H
\end{array}\right) \geq 0, \\
& \left(\begin{array}{ll}
X & I \\
I & M
\end{array}\right) \geq 0, \\
& \left(\begin{array}{cc}
J & I \\
I & F
\end{array}\right) \geq 0, \\
& \left(\begin{array}{cccc}
\widehat{\varphi}_{8 \times 8} & 0 & \Lambda_{3}^{T} & \Gamma_{1}^{T} \\
\star & -\widehat{S} & -\widehat{S} & 0 \\
\star & \star & -J & 0 \\
\star & \star & \star & -N
\end{array}\right)<0 .
\end{aligned}
$$

If the solution of the above minimization problem is $3 n$, that is, $\operatorname{tr}(N H+X M+J F)=3 n$, we can say from Theorem 8 that system (1) with the control $u(t)=L X^{-1} x(t)$ is asymptotically stable. Although it is still impossible to always find the global optimal solution, the proposed nonlinear minimization problem is easier to solve than the original nonconvex feasibility problem. Actually, utilizing the linearization method [30], we can easily find a suboptimal maximal delay using an iterative algorithm presented in the following.

Note that condition (29) is used as a stopping criterion in the algorithm since it is numerically very difficult in practice to obtain the optimal solution such that $\operatorname{tr}(\mathrm{NH}+\mathrm{XM}+J F)$ is exactly equal to $3 n$.

The above calculation thought can be carried out by the following steps.

\section{Algorithm 9.}

Step 1. Choose a sufficiently small initial integer $\bar{n}>0$ such that there exists a feasible solution to the LMI conditions in (37) and (38). Set $\bar{n}_{\text {so }}=\bar{n}$.

Step 2. Find a feasible set

$$
\begin{aligned}
& \left\{X_{0}, \widehat{W}_{10}, \widehat{W}_{20}, \widehat{Q}_{10}, \widehat{Q}_{20}, \widehat{R}_{10}, \widehat{R}_{20}, \widehat{Y}_{0}, \widehat{Z}_{0}, \widehat{R}_{0}, L_{0}, H_{0}, M_{0},\right. \\
& \left.\quad F_{0}, N_{0}, J_{0}\right\}
\end{aligned}
$$

for LMIs (37)-(39). Set $k=0$, where

$$
\widehat{R}_{0}=\left(\begin{array}{cc}
\widehat{R}_{110} & \widehat{R}_{120} \\
\star & \widehat{R}_{220}
\end{array}\right) .
$$

Step 3. Solve the following LMI problem for the variables: 


$$
\begin{aligned}
& \left\{X, \widehat{W}_{1}, \widehat{W}_{2}, \widehat{Q}_{1}, \widehat{Q}_{2}, \widehat{R}_{1}, \widehat{R}, \widehat{Y}, \widehat{Z}, \widehat{R}, L, H, M, F, N, J\right\}: \\
\text { Minimize } & \operatorname{Trace}\left(N_{k} H+H_{k} N+X_{k} M+M_{k} X+J_{k} F+F_{k} J\right) \\
\text { subject to } & \text { (37)-(39). }
\end{aligned}
$$

Set $N_{k+1}=N, H_{k+1}=H, X_{k+1}=X, M_{k+1}=M, J_{k+1}=J$, and $F_{k+1}=F$.

Step 4. If condition (29) is satisfied, then set $\bar{h}_{\mathrm{so}}=\bar{h}$ and return to Step 2 after increasing $\bar{h}$ to some extent. If condition (29) is not satisfied within a specified number of iterations, say, $k_{\max }$, then exit. Otherwise, set $k=k+1$ and go to Step 3.

The above algorithm gives a suboptimal maximal delay $\bar{h}_{\text {so }}$ such that system (1) can be stabilized with the controller $u(t)=L X^{-1} x(t)$. Later, in Section 4, we shall illustrate via numerical examples that the above algorithm can provide quite satisfactory results.

Similar to Theorem 8, the stabilization condition for system (1) with $\tau=h$ can be easily obtained.

Theorem 10. System (1) with $\tau=h$ is asymptotically stabilized if there exist positive definite matrices $X, \widehat{W}_{2}, \widehat{Q}_{2}, \widehat{R}_{2}$, symmetric matrices $\widehat{Y}_{i i}, \widehat{Z}_{i i}(i=1,2,3), \widehat{R}_{11}, \widehat{R}_{22}$, and any matrices $\widehat{Y}_{i j}, \widehat{Z}_{i j}(i \neq j \in 1,2,3), \widehat{R}_{12}$, L with appropriate dimensions satisfying the following linear matrix inequalities:

$$
\left(\begin{array}{cccc}
\widehat{\varphi}_{5 \times 5} & 0 & \Lambda_{4}^{T} & \Gamma_{2}^{T} \\
\star & -\widehat{S} & \widehat{S} & 0 \\
\star & \star & -J & 0 \\
\star & \star & \star & -X J^{-1} X
\end{array}\right)<0
$$

where

$$
\begin{aligned}
& \widehat{\Psi}_{11}=X+\widehat{Y}_{11}+\widehat{Z}_{11}+6 h \widehat{W}_{2}+\frac{4}{h} \widehat{R}_{22}+\frac{3 h}{2} \widehat{R}_{2}, \\
& \widehat{\Psi}_{12}=\widehat{Y}_{12}+\widehat{Z}_{12}+\frac{2}{h} \widehat{R}_{22}, \\
& \widehat{\Psi}_{13}=6 h \widehat{W}_{2}-2 \widehat{R}_{12}^{T}+h \widehat{Y}_{13}-\frac{6}{h} \widehat{R}_{22}, \\
& \widehat{\Psi}_{14}=6 \widehat{R}_{12}^{T}-24 h \widehat{W}_{2}+h^{2} \widehat{Z}_{13}-\frac{3 h}{2} \widehat{R}_{2},
\end{aligned}
$$

$$
\begin{aligned}
& \widehat{\Psi}_{22}=\widehat{Y}_{22}+\widehat{Z}_{22}+\frac{4}{h} \widehat{R}_{22} \text {, } \\
& \widehat{\Psi}_{23}=h \widehat{Y}_{23}-4 \widehat{R}_{12}^{T}-\frac{6}{h} \widehat{R}_{22} \text {, } \\
& \widehat{\Psi}_{24}=h^{2} \widehat{Z}_{23}+6 \widehat{R}_{12}^{T} \text {, } \\
& \widehat{\Psi}_{33}=4 h \widehat{Q}_{2}+18 h \widehat{W}_{2}+6 \widehat{R}_{12}+6 \widehat{R}_{12}^{T}+4 h \widehat{R}_{11} \\
& +\frac{12}{h} \widehat{R}_{22}+h^{2} \widehat{Y}_{33} \\
& \widehat{\Psi}_{34}=-6 h \widehat{Q}_{2}-48 h \widehat{W}_{2}-12 \widehat{R}_{12}^{T}-6 h \widehat{R}_{11} \text {, } \\
& \widehat{\Psi}_{44}=12 h \widehat{Q}_{2}+144 h \widehat{W}_{2}+12 h \widehat{R}_{11}+6 h \widehat{R}_{2}+h^{4} \widehat{Z}_{33} \text {, } \\
& \Lambda_{4}=\left[\begin{array}{lllll}
\widehat{Y}_{11}+\widehat{Z}_{11}+\widehat{R}_{12}^{T} & \widehat{Y}_{12}+\widehat{Z}_{12} & 0 & \widehat{Y}_{13} & \widehat{Z}_{13}
\end{array}\right], \\
& \Gamma_{2}=\left[\begin{array}{llllll}
A X+D L & B X & C X & 0 & 0 & 0
\end{array}\right], \\
& \widehat{\varphi}_{11}=\widehat{R}_{11}-6 \widehat{R}_{2}-9 \widehat{W}_{2}+\widehat{Y}_{13}+\widehat{Y}_{13}^{T}+h \widehat{Z}_{13}+h \widehat{Z}_{13}^{T} \\
& +X A^{T}+A X+D L+L^{T} D^{T}+\widehat{Q}_{2} \text {, } \\
& \widehat{\varphi}_{12}=3 \widehat{W}_{2}-\widehat{Y}_{13}+\widehat{Y}_{23}^{T}+h \widehat{Z}_{23}^{T}+B X, \\
& \widehat{\varphi}_{13}=\widehat{Y}_{12}+\widehat{Z}_{12}+C X \text {, } \\
& \widehat{\varphi}_{14}=h \widehat{Y}_{33}-24 \widehat{W}_{2}-h \widehat{Z}_{13}-6 \widehat{R}_{2} \text {, } \\
& \widehat{\varphi}_{15}=24 \widehat{R}_{2}+60 \widehat{W}_{2}+h^{3} \widehat{Z}_{33} \text {, } \\
& \widehat{\varphi}_{22}=-\widehat{R}_{11}-9 \widehat{W}_{2}-\widehat{Y}_{23}-\widehat{Y}_{23}^{T}-\widehat{Q}_{2}, \\
& \widehat{\varphi}_{23}=\widehat{Y}_{22}-\widehat{R}_{12}+\widehat{Z}_{22} \text {, } \\
& \widehat{\varphi}_{24}=36 \widehat{W}_{2}-h \widehat{Z}_{23}-h \widehat{Y}_{33} \text {, } \\
& \widehat{\varphi}_{25}=-60 \widehat{W}_{2}, \\
& \widehat{\varphi}_{33}=-\widehat{R}_{22}, \\
& \widehat{\varphi}_{34}=h \widehat{Y}_{23} \text {, } \\
& \widehat{\varphi}_{35}=h^{2} \widehat{Z}_{23} \text {, } \\
& \widehat{\varphi}_{44}=-18 \widehat{R}_{2}-192 \widehat{W}_{2} \text {, } \\
& \widehat{\varphi}_{45}=48 \widehat{R}_{2}+360 \widehat{W}_{2}-h^{3} \widehat{Z}_{33} \text {, }
\end{aligned}
$$


TABLE 1: The maximum of $h$ for different $\tau$.

\begin{tabular}{lccc}
\hline References & $\tau=0.1$ & $\tau=0.5$ & $\tau=1.0$ \\
\hline$[7]$ & 1.7100 & 1.6718 & 1.6543 \\
{$[8]$} & 1.7844 & 1.7495 & 1.7201 \\
{$[17]$} & 1.8307 & 1.7755 & 1.7213 \\
{$[5]$} & 2.1229 & 2.1229 & 2.1229 \\
{$[6]$} & 2.2951 & 2.3471 & 2.3752 \\
Theorem 3 $(N=1)$ in [9] & 1.8413 & 2.0954 & 2.2611 \\
Theorem 3 $(N=3)$ in [9] & 2.1845 & 2.2973 & 2.3331 \\
Theorem 3 $(N=5)$ in [9] & 2.2137 & 2.3210 & 2.3588 \\
Theorem 1 in [4] & 2.2021 & 2.2469 & 2.2670 \\
Theorem 2 $(N=1)$ in [4] & 1.8432 & 2.1078 & 2.2587 \\
Theorem 2 $(N=3)$ in [4] & 2.1886 & 2.3100 & 2.3452 \\
Theorem 2 $(N=5)$ in [4] & 2.2181 & 2.3331 & 2.3636 \\
Theorem 4 & 2.2959 & 2.3488 & 2.3769 \\
Analytical bounds & 2.2963 & 2.3491 & 2.3775 \\
\hline
\end{tabular}

$$
\begin{aligned}
\widehat{\varphi}_{55} & =-144 \widehat{R}_{2}-720 \widehat{W}_{2}, \\
\widehat{S} & =\widehat{R}_{22}+\frac{h^{2}}{2} \widehat{R}_{2}+h^{2} \widehat{W}_{2},
\end{aligned}
$$

and the state feedback controller is $u(t)=L X^{-1} x(t)$.

\section{Examples}

In this section, we will demonstrate the superiority of our approach in the above section using the conventional neutral system by the following examples.

Example 1 (see [1]). Consider system (1) with the state matrices listed as follows:

$$
\begin{aligned}
& A=\left(\begin{array}{cc}
-0.9 & 0.2 \\
0.1 & -0.9
\end{array}\right), \\
& B=\left(\begin{array}{cc}
-1.1 & -0.2 \\
-0.1 & -1.1
\end{array}\right), \\
& C=\left(\begin{array}{cc}
-0.2 & 0 \\
0.2 & -0.1
\end{array}\right) .
\end{aligned}
$$

With different cases, we can obtain different maximum time delay $h$ by using Matlab toolbox [25] as listed in Tables 1 and 2, compared with some existing references.

The first case is $\tau \neq h$, and the maximum of $h$ for different $\tau$ compared to the existing results are listed in Table 1. From Table 1, we can see that the results in $[4,9]$ are close to our results. However, the maximum of $h$ using Theorem 4 is closer to analytical bounds, which shows our approach is less conservative than the existing results. It is shown that we may also obtain better delay-dependent stability condition without using discrete Lyapunov functional approach such as in [6].
TABLE 2: The maximum of $h$ as $h=\tau$.

\begin{tabular}{lc}
\hline References & $\tau=0.1$ \\
\hline$[10]$ & 1.3718 \\
{$[7]$} & 1.6527 \\
{$[20]$} & 1.7191 \\
{$[21]$} & 1.7220 \\
{$[8]$} & 1.7844 \\
{$[23]$} & 1.7856 \\
{$[17]$} & 1.8307 \\
{$[5]$} & 2.2254 \\
Theorem $3(N=5)[9]$ & $2.2069\left(N_{v}=113\right)$ \\
Theorem 3 $(N=5)[4]$ & $2.2069\left(N_{v}=65\right)$ \\
Theorem 4 & $2.2250\left(N_{v}=60\right)$ \\
Analytical bounds & 2.2255 \\
\hline
\end{tabular}

The second case is $\tau=h$, and the maximum of $h$ compared to the existing results are listed in Table 2, where $N_{v}$ denotes the $N$ of variables to compute the LMIs of stability conditions. Although our result is a little less than that in [5], our condition is the best result except the condition obtained by using the approach in [5]. Furthermore, our approach may bring simpler computation than that in [5], because the $N$ of variables to compute the maximum delay are $13 n^{2}+6 n$ in [5] and $12 n^{2}+6 n$ in this paper, respectively. From the comparison between [4] and [9], [4] improves the results in [9] on the computation of variables. However, our results not only decrease the number of variables but also increase the maximum bound of delay compared to [4]. Furthermore, using our approach may be more efficient to deal with neutral systems with mixed time-varying delay.

In a word, it is observed that our method presented in this paper is less conservative than most of the existing results.

In order to widely show the effectiveness and superiority of our approach presented in this paper, the next examples should be listed.

Example 2 (see [1]). Consider system (1) with the state matrices listed as follows:

$$
\begin{aligned}
& A=\left(\begin{array}{cc}
-2 & 0 \\
0 & -0.9
\end{array}\right), \\
& B=\left(\begin{array}{cc}
-1 & 0 \\
-1 & -1
\end{array}\right), \\
& C=\left(\begin{array}{ll}
c & 0 \\
0 & c
\end{array}\right),
\end{aligned}
$$

with the positive scalar $c$ and $\tau=h$. With different $c$, we can obtain different maximum time delay $h$ by using Matlab toolbox [25] as listed in Table 3, compared with the existing references. For the sake of simplicity, the computation for the $N$ of variables in $[1-3,18,19]$ is omitted here.

Clearly, the results of $h$ in $[1-3,18,19]$ are much more conservative than those in $[12,13]$ and Theorem 7 . It is 
TABLE 3: The maximal allowable delays $h$ of Example 2 for different values of $c$.

\begin{tabular}{|c|c|c|c|c|c|}
\hline$c$ & 0 & 0.1 & 0.3 & 0.5 & $N$ of variables \\
\hline [1] & 4.47 & 3.49 & 2.06 & 1.14 & Omitted \\
\hline$[18]$ & 4.35 & 4.33 & 4.10 & 3.62 & Omitted \\
\hline [2] & 4.47 & 4.35 & 4.13 & 3.67 & Omitted \\
\hline [3] & 4.47 & 4.42 & 4.17 & 3.69 & Omitted \\
\hline [19] & 4.63 & 4.57 & 4.29 & 3.75 & Omitted \\
\hline$[13]$ & 5.30 & 5.21 & 4.85 & 4.20 & $6 n^{2}+3.5 n$ \\
\hline Theorem $1(N=6)[12]$ & 5.8613 & 5.7781 & 5.3946 & 4.6687 & $46 n^{2}+11 n$ \\
\hline Theorem $1(N=7)[12]$ & 5.8779 & 5.7910 & 5.4007 & 4.6703 & $56.5 n^{2}+12.5 n$ \\
\hline Theorem $1(N=8)[12]$ & 5.9287 & 5.8427 & 5.4417 & 4.6917 & $68 n^{2}+14 n$ \\
\hline Theorem $1(N=9)[12]$ & 6.1028 & 5.9816 & 5.5173 & 4.7247 & $80.5 n^{2}+15.5 n$ \\
\hline$[28]$ & 6.117 & - & - & - & - \\
\hline Theorem 7 & 6.1689 & 6.0339 & 5.5470 & 4.7374 & $12 n^{2}+6 n$ \\
\hline
\end{tabular}

observed from Table 3 that our time delay $h$ is much bigger than the existing results with the less variables.

This example shows again that our approach is effective and less conservative than the existing results.

Example 3. Consider the PEEC model introduced in $[24,32]$ with $\tau=h$ and the matrices parameters listed as follows:

$$
\begin{aligned}
& A=100 \times\left(\begin{array}{ccc}
\theta & 1 & 2 \\
3 & -9 & 0 \\
1 & 2 & -6
\end{array}\right), \\
& B=100 \times\left(\begin{array}{ccc}
1 & 0 & -3 \\
-0.5 & -0.5 & -1 \\
-0.5 & -1.5 & 0
\end{array}\right), \\
& C=\frac{1}{72} \times\left(\begin{array}{ccc}
-1 & 5 & 2 \\
4 & 0 & 3 \\
-2 & 4 & 1
\end{array}\right) .
\end{aligned}
$$

With our approach and using Matlab toolbox [25], the maximum time delay $h$ compared to the existing results is listed in Table 4. It is quite evident that our result is the best.

Example 4 (see [3]). Consider the neutral system (1) with $\tau=$ $h$ as the following parameters:

$$
\begin{aligned}
& A=\left(\begin{array}{cc}
-1.7073 & 0.6856 \\
0.2279 & -0.6368
\end{array}\right), \\
& B=\left(\begin{array}{cc}
-2.5026 & -1.0540 \\
-0.1856 & -1.5715
\end{array}\right), \\
& C=\left(\begin{array}{ll}
0.0558 & 0.0360 \\
0.2747 & -0.1084
\end{array}\right) .
\end{aligned}
$$

Using the conditions in [7], [3], [21], and [2] and with Matlab toolbox [25], the maximum values of time delay $h$ are calculated as $0.5735,0.5937,0.6054$, and 0.6189 , respectively.
TABLE 4: The maximal allowable delays $h$ for different values of $\theta$.

\begin{tabular}{lccc}
\hline$\theta$ & -2.105 & -2.103 & -2.1 \\
\hline$[31]$ & 1.0874 & 0.3709 & 0.2433 \\
{$[32]$} & 1.1413 & 0.3892 & 0.2533 \\
Theorems 1 and 2 in [33] & 1.3200 & 0.4917 & 0.3214 \\
Theorems 3 and 4 in [33] & 1.6978 & 0.5747 & 0.3749 \\
Theorem 7 & 1.7824 & 0.6030 & 0.3930 \\
\hline
\end{tabular}

Now applying our approach in this paper, the maximum time delay $h$ is computed as 0.7029 . It is $22.56,18.39,16.11$, and 13.57 larger than those in [7], [3], [21], and [2], respectively.

On the other hand, the neutral system is changed into delay system as $C=0$, and the maximum of $h$ is obtained as 0.6093 in $[1,22,36]$. Although the maximum of $h$ can reach 0.7163 and 0.7198 in [3] and [2], respectively, our result is 0.9351 which is $30.55 \%$ and $30.07 \%$ larger than those in [3] and [2]. This example shows again that our conditions are less conservative than the existing results.

Example 5 (see [13]). Consider the following linear timedelay control system:

$$
\dot{x}(t)-C \dot{x}(t-\tau)=A x(t)+B x(t-h)+D u(t),
$$

where

$$
\begin{aligned}
A & =\left(\begin{array}{ll}
0 & 0 \\
0 & 1
\end{array}\right), \\
B & =\left(\begin{array}{cc}
-1 & -1 \\
0 & -0.9
\end{array}\right), \\
C & =\left(\begin{array}{ll}
0 & 0 \\
0 & 0
\end{array}\right), \\
D & =\left(\begin{array}{l}
0 \\
1
\end{array}\right) .
\end{aligned}
$$


TABLE 5: The comparison of different controller design methods for $h$.

\begin{tabular}{|c|c|c|c|c|}
\hline Method & $h$ & & $K$ & Iterations \\
\hline [34] & 0.6779 & {$[-0.1155$} & $-1.9839]$ & - \\
\hline [35] & 1.51 & {$[-58.31$} & -294.935] & - \\
\hline [37] & 1.51 & {$[-0.7851$} & $-2.0379]$ & 6 \\
\hline [37] & 8 & {$[-65.4058$} & $-76.7778]$ & 111 \\
\hline [13] & 9 & {$[-44.1358$} & $-49.0181]$ & 94 \\
\hline [13] & 10 & {$[-86.3203$} & $-93.8552]$ & 164 \\
\hline [13] & 11 & {$[-153.1753$} & $-164.7362]$ & 247 \\
\hline Theorem 10 & 9 & {$[-1.4897$} & $-3.0566]$ & 34 \\
\hline Theorem 10 & 10 & {$[-1.8289$} & $-3.4439]$ & 31 \\
\hline Theorem 10 & 11 & {$[-2.2411$} & -3.9037] & 34 \\
\hline Theorem 10 & 15 & {$[-4.1009$} & $-5.9452]$ & 39 \\
\hline Theorem 10 & 25 & {$[-9.2669$} & $-11.2516]$ & 43 \\
\hline
\end{tabular}

With the help of Matlab toolbox [25], Table 5 presents the delay bounds and feedback gain matrices obtained in $[13,34$, 35,37 ] along with those achieved in this paper.

From the comparison in Table 5, we can see that our approach needs less iterations to design the feedback controller for the same delay $h$. Furthermore, the proposed stabilization criteria obtained in this paper provide a significantly better upper delay bound than that given in $[13,34,35,37]$.

\section{Conclusion}

In this paper, the delay-dependent stabilization problems for neutral systems have been investigated based on the improved Jensen's inequalities and new Lyapunov-Krasovskii functionals. Based on a new class of constructed LyapunovKrasovskii functionals and combined with the new integral inequalities, two new stability criteria have been firstly obtained for neutral systems with mixed time delay. Then, state feedback controllers have been designed using a cone complementarity linearization method for the neutral control systems in different cases. Finally, five examples have shown the effectiveness and less conservativeness of the condition presented in this paper. Our future work is to obtain more efficient and less conservative stabilization conditions for neutral dynamical systems with time-varying delay by using our new technique presented in this paper.

\section{Competing Interests}

The authors declare that they have no competing interests.

\section{Acknowledgments}

This work is supported by National Nature Science Foundation of China under Grants 11461082, 11601474, and 61472093 and Scientific Research Fund Project in Yunnan Provincial Department of Education under Grants 2015J069 and 2015 Y223.

\section{References}

[1] E. Fridman and U. Shaked, "Delay-dependent stability and $H_{\infty}$ control: constant and timevarying delays," International Journal of Control, vol. 76, no. 1, pp. 48-60, 2003.

[2] M. Wu, Y. He, and J.-H. She, "New delay-dependent stability criteria and stabilizing method for neutral systems," IEEE Transactions on Automatic Control, vol. 49, no. 12, pp. 22662271, 2004

[3] Y. He, Q.-G. Wang, C. Lin, and M. Wu, "Augmented Lyapunov functional and delay-dependent stability criteria for neutral systems," International Journal of Robust and Nonlinear Control, vol. 15, no. 18, pp. 923-933, 2005.

[4] L. Ding, Y. He, M. Wu, and C. Ning, "Improved mixed-delaydependent asymptotic stability criteria for neutral systems," IET Control Theory \& Applications, vol. 9, no. 14, pp. 2180-2187, 2015.

[5] Q.-L. Han, "On stability of linear neutral systems with mixed time delays: a discretized Lyapunov functional approach," Automatica, vol. 41, no. 7, pp. 1209-1218, 2005.

[6] X.-G. Li, X.-J. Zhu, A. Cela, and A. Reama, "Stability analysis of neutral systems with mixed delays," Automatica, vol. 44, no. 11, pp. 2968-2972, 2008.

[7] Y. He, M. Wu, J.-H. She, and G.-P. Liu, "Delay-dependent robust stability criteria for uncertain neutral systems with mixed delays," Systems \& Control Letters, vol. 51, no. 1, pp. 57-65, 2004.

[8] X.-G. Liu, M. Wu, R. Martin, and M.-L. Tang, "Stability analysis for neutral systems with mixed delays," Journal of Computational and Applied Mathematics, vol. 202, no. 2, pp. 478-497, 2007.

[9] Y. Chen, S. Fei, Z. Gu, and Y. Li, "New mixed-delay-dependent robust stability conditions for uncertain linear neutral systems," IET Control Theory \& Applications, vol. 8, no. 8, pp. 606-613, 2014.

[10] J. H. Park and O. Kwon, "On new stability criterion for delaydifferential systems of neutral type," Applied Mathematics and Computation, vol. 162, no. 2, pp. 627-637, 2005.

[11] A. Seuret, F. Gouaisbaut, and E. Fridman, "Stability of systems with fast-varying delay using improved Wirtinger's inequality," in Proceedings of the 52nd IEEE Conference on Decision and Control (CDC '13), pp. 946-951, IEEE, Florence, Italy, December 2013.

[12] M. Fang and J. H. Park, "A multiple integral approach to stability of neutral time-delay systems," Applied Mathematics and Computation, vol. 224, no. 1, pp. 714-718, 2013.

[13] J. Sun, G. P. Liu, and J. Chen, "Delay-dependent stability and stabilization of neutral time-delay systems," International Journal of Robust and Nonlinear Control, vol. 19, no. 12, pp. 13641375, 2009.

[14] M. Park, O. Kwon, J. H. Park, S. Lee, and E. Cha, "Stability of time-delay systems via Wirtinger-based double integral inequality," Automatica, vol. 55, pp. 204-208, 2015.

[15] H.-B. Zeng, Y. He, M. Wu, and J. She, "New results on stability analysis for systems with discrete distributed delay," Automatica, vol. 60, pp. 189-192, 2015.

[16] H.-B. Zeng, Y. He, M. Wu, and J. She, "Free-matrix-based integral inequality for stability analysis of systems with timevarying delay," IEEE Transactions on Automatic Control, vol. 60, no. 10, pp. 2768-2772, 2015.

[17] W. Qian, J. Liu, Y. Sun, and S. Fei, "A less conservative robust stability criteria for uncertain neutral systems with mixed delays," Mathematics and Computers in Simulation, vol. 80, no. 5, pp. 1007-1017, 2010. 
[18] Q.-L. Han, "Robust stability of uncertain delay-differential systems of neutral type," Automatica, vol. 38, no. 4, pp. 719-723, 2002.

[19] M. N. Parlakci, "Robust stability of uncertain neutral systems: a novel augmented Lyapunov functional approach," IET Control Theory \& Applications, vol. 1, no. 3, pp. 802-809, 2007.

[20] O. M. Kwon, J. H. Park, and S. M. Lee, “On stability criteria for uncertain delay-differential systems of neutral type with timevarying delays," Applied Mathematics and Computation, vol. 197, no. 2, pp. 864-873, 2008.

[21] S. Xu, J. Lam, and Y. Zou, "Further results on delay-dependent robust stability conditions of uncertain neutral systems," International Journal of Robust and Nonlinear Control, vol. 15, no. 5, pp. 233-246, 2005.

[22] S. Xu and J. Lam, "Improved delay-dependent stability criteria for time-delay systems," IEEE Transactions on Automatic Control, vol. 50, no. 3, pp. 384-387, 2005.

[23] Z. Zhao, W. Wang, and B. Yang, "Delay and its time-derivative dependent robust stability of neutral control system," Applied Mathematics and Computation, vol. 187, no. 2, pp. 1326-1332, 2007.

[24] J. K. Hale, Theory of Functional Differential Equations, Springer, New York, NY, USA, 2nd edition, 1977.

[25] L. Yu, Robust Control: A LMI Approach, Tsinghua University Press, 2002.

[26] H. Chen, P. Hu, and J. Wang, "Delay-dependent exponential stability for neutral stochastic system with multiple time-varying delays," IET Control Theory and Applications, vol. 8, no. 17, pp. 2092-2101, 2014.

[27] P. Park, W. I. Lee, and S. Y. Lee, "Auxiliary function-based integral inequalities for quadratic functions and their applications to time-delay systems," Journal of the Franklin Institute, vol. 352, no. 4, pp. 1378-1396, 2015.

[28] C.-Y. Kao and A. Rantzer, "Stability analysis of systems with uncertain time-varying delays," Automatica, vol. 43, no. 6, pp. 959-970, 2007.

[29] H. Gao and C. Wang, "Comments and further results on, a descriptor system approach to $H_{\infty}$ control of linear time-delay systems," IEEE Transaction on Automatic Control, vol. 48, no. 3 , pp. 520-525, 2003.

[30] L. El Ghaoui, F. Oustry, and M. Aitrami, "A cone complementarity linearization algorithm for static output-feedback and related problems," IEEE Transactions on Automatic Control, vol. 42, no. 8, pp. 1171-1176, 1997.

[31] Q.-L. Han, "On robust stability of neutral systems with timevarying discrete delay and norm-bounded uncertainty," Automatica, vol. 40, no. 6, pp. 1087-1092, 2004.

[32] D. Yue and Q.-L. Han, "A delay-dependent stability criterion of neutral systems and its application to a partial element equivalent circuit model," IEEE Transactions on Circuits and Systems II: Express Briefs, vol. 51, no. 12, pp. 685-689, 2004.

[33] J. Sun and G. P. Liu, "On improved delay-dependent stability criteria for neutral time-delay systems," European Journal of Control, vol. 15, no. 6, pp. 613-623, 2009.

[34] X. Li and C. E. de Souza, "Delay-dependent robust stability and stabilization of uncertain linear delay systems: a linear matrix inequality approach," IEEE Transactions on Automatic Control, vol. 42, no. 8, pp. 1144-1148, 1997.

[35] E. Fridman and U. Shaked, "An improved stabilization method for linear time-delay systems," IEEE Transactions on Automatic Control, vol. 47, no. 11, pp. 1931-1937, 2002.
[36] V. Suplin, E. Fridman, and U. Shaked, " $H_{\infty}$ control of linear uncertain time-delay systems-a projection approach," IEEE Transactions on Automatic Control, vol. 51, no. 4, pp. 680-685, 2006.

[37] M. N. Parlakçı, "Improved robust stability criteria and design of robust stabilizing controller for uncertain linear time-delay systems," International Journal of Robust and Nonlinear Control, vol. 16, no. 13, pp. 599-636, 2006. 


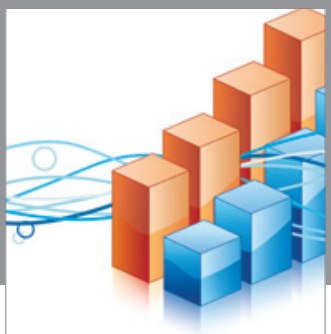

Advances in

Operations Research

vatem alat4

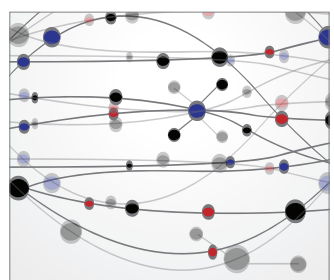

\section{The Scientific} World Journal
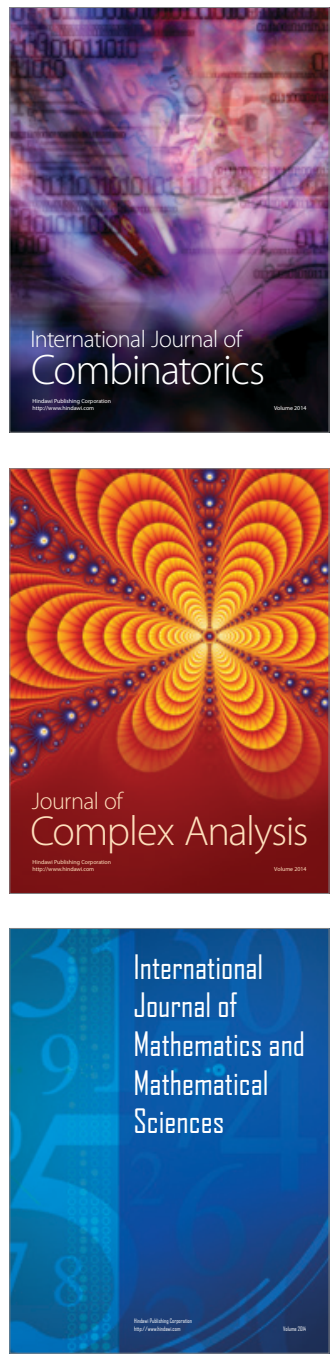
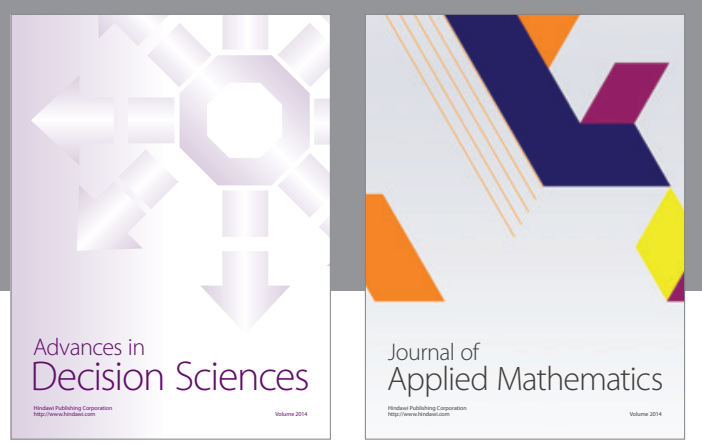

Algebra

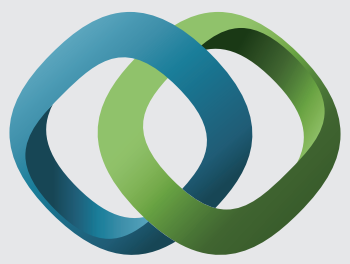

\section{Hindawi}

Submit your manuscripts at

http://www.hindawi.com
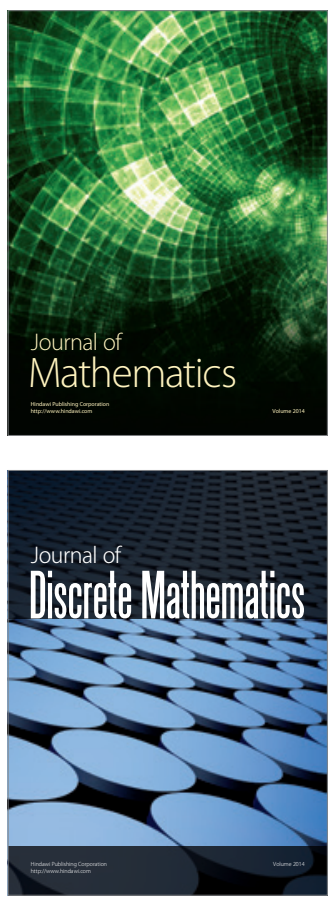

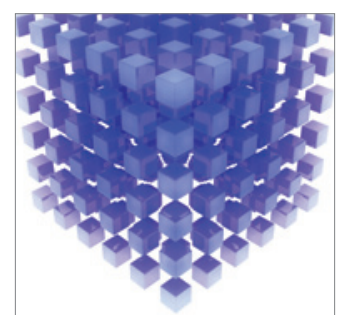

Mathematical Problems in Engineering
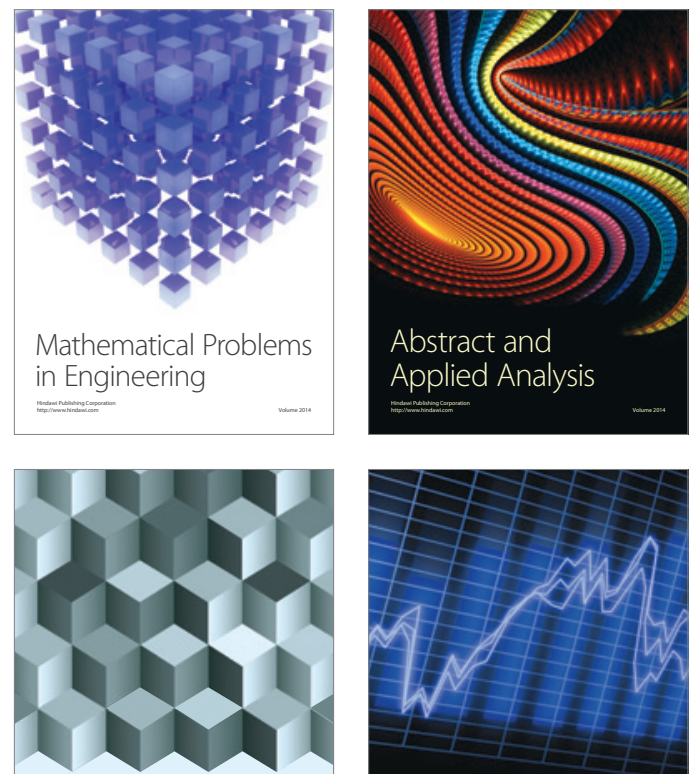

Journal of

Function Spaces

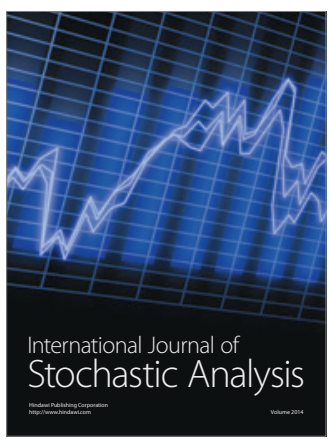

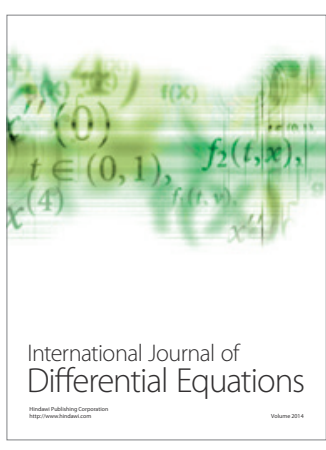
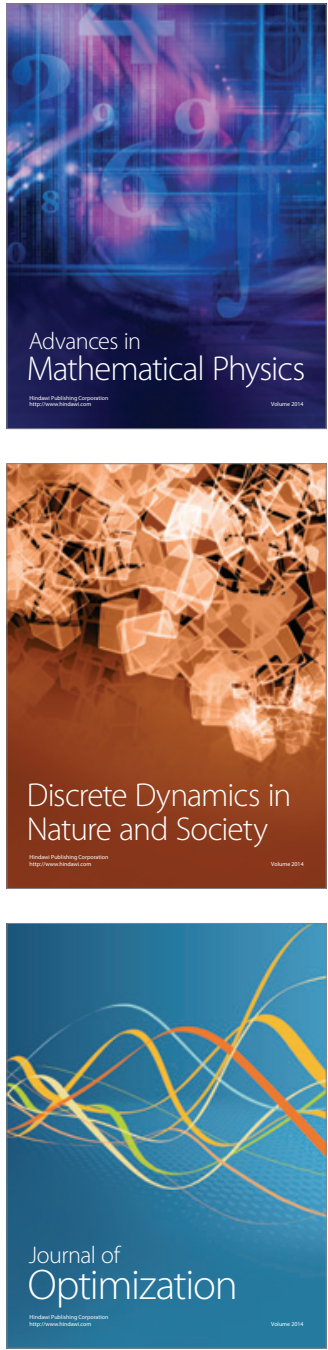